\title{
1. Disentangling the mechanistic chain for better policy design
}

\section{Giliberto Capano, Michael Howlett and M Ramesh}

\section{A MECHANISTIC TURN FOR POLICY DESIGN?}

Understanding how policy design can incentivize, constrain, and otherwise structure policy targets' behavior to achieve desired results is vital but requires a clear understanding of the mechanisms that link policy tools to actual behavior. More importantly, it requires reasoning in terms of the processes and interactions that can be activated by policy tools to accomplish desired results. It is therefore imperative that policy designers - both those who study it and those who practice it - specify clearly the linkages between the input (policy design) and the output, via the intended and unintended processes triggered by the design. Many existing analytical efforts focus only on shedding light on what is needed for good policy design and ignore how good policy design works in terms of the types of processes that can be activated to achieve (or not) the desired results. As a result, we know little about how different solutions trigger and drive the achievement of intended outcomes. The literature on policy design is often based on anecdotes and correlations, jumping from proposed solutions to anticipated outcomes without exploring the conditions that are the real determinants of policy results.

The objective of this book is to explore the usefulness of adopting a mechanistic approach to policy design, focusing on the actual ways in which policy designs can facilitate or hinder achievement of policy goals. It improves the analysis and practice of policy design by focusing on the mechanistic causation relevant to policy-making and policy behavior. The book thus brings to policy design studies the insights of the mechanistic turn in social sciences over the past few decades. This mechanistic turn is partly motivated by dissatisfaction with both the "law-like" and statistical explanations commonly employed by policy scholars. 
Starting with Elster (1989), many studies in both political science and sociology have sought to apply a mechanistic explanatory logic (Hedström and Swedberg, 1998; Tilly, 2001; Maynitz, 2004; Schmid, 2006; Abbott, 2007; Gross, 2009). While the mechanistic line of thinking has found a ready home in sociology, the idea of causal mechanisms remains controversial in political science. As has been observed, there are many definitions of mechanisms (Mahoney, 2001; Gerring, 2007), leading to the questioning of its ontological status (Gerring, 2010 vs Falleti and Lynch, 2009). The debate remains inconclusive, with diverging opinions on the role of mechanisms and mechanistic explanations based on different epistemological views.

Although there is general agreement that mechanisms are "analytical constructs" that explain observed behavior (Hedström and Swedberg, 1998, p. 13), mechanisms have been defined in multiple ways. Kuorikoski (2009) has summarized the debate by proposing two ways to conceptualize mechanisms: as an "abstract form of interaction" (AFI) or "componential causal system" (CCS).

AFI conceptualizes mechanisms as an event or activity that can have different causes and exhibits a specific form of causal interaction. Falleti and Lynch (2009) define mechanisms as "portable concepts that describe how causation occurs" (p. 1148) and thus "tell us how things happen: how actors relate, how individuals come to believe what they do or what they draw from past experience, how policies and institutions endure or change" (p. 1147). All these explanations are based on a specific context. AFI, which is the definition most frequently adopted in social sciences, considers mechanisms in a largely processual and contextual manner. According to this perspective, mechanisms are abstract concepts (which may be unobservable) that can explain a phenomenon given a specific context and characteristic process through which a specific result is achieved. For example, Tilly notes that mechanisms "form a delimited class of events that change relations among specified sets of elements in identical or closely similar ways over a variety of situations" (Tilly, 2001, pp. 25-6). Gerring defines a mechanism as "the pathway or process by which an effect is produced or a purpose is accomplished" (Gerring, 2007, p. 178).

The "componential causal system" approach, on the other hand, assumes that mechanisms are not only processes but also have a structure in the sense that they are composed of entities with properties and activities of their own. A mechanism, accordingly, is a type of "systemic" phenomenon that bridges cause and effect but is also "a structure performing a function in virtue of its component parts, component operations, and their organization. The orchestrated functioning of mechanism is responsible for one or more phenomena" (Bechtel and Abrahamsen, 2005, p. 423). As Glennan (2011, p. 809) notes, mechanisms are "particular systems of interacting parts, where these interactions occur at a particular time and place." Here, the concept of mechanism is more complex than with AFI: it is not only process but can also be a complex 
structure of organized parts. More specifically, according to this perspective, a mechanism can be defined as "a constellation of entities and activities that are organized such that they regularly bring about a particular type of outcome" (Hedström, 2008, p. 321). From this perspective, a "consensus" definition describes a mechanism as a "system with multiple components, which interact to produce some overall phenomenon" (Fagan, 2012, p. 453; see also Bechtel and Abrahamsen, 2005). When activated, such systems "actualize" potential powers, engendering both local and general effects.

Hence, the (AFI) perspective on mechanisms is focused on identifying "the intervening causal process - the causal chain and the causal mechanisms - between an independent variable (or variables) and the outcome of the dependent variable" (George and Bennett, 2005, pp. 206-7). From the systemic perspective (CCS), however, a mechanism is a specific configuration of elements that is capable of producing a specific process (or behavior) in a specific context. In other words, mechanisms should be considered as recurrent patterns of the interaction of invariant change-producing elements.

From a policy design perspective, both approaches are useful. The AFI's conception of mechanisms, for example, can be useful for understanding policy dynamics by focusing attention on those "abstracted" factors through which effects are produced in a specific policy context, such as the psychological mechanisms through which various "nudges" operate (Capano, 2013). The CCS's conception of mechanisms as a system of interacting parts, on the other hand, helps better understand policy-making institutions and processes such as the impact of electoral systems or legislative activities.

Regardless of how it is defined, a mechanistic approach is useful for policy analysis and policy design for several reasons. First, it points to the idea that to understand what happens, and thus to design something to happen, policy designers must pay attention to and theorize not only what is observable - such as behavior of actors or networks - but also what may be unobservable, such as the root causes of that behavior.

Second, it highlights that in any design, the designed policy must impact the mechanistic workings of the actual situation if it is to have any effect. Thus: (1) if the AFI definition of mechanism is adopted, policy design should be capable of activating the proper mechanism(s); (2) if the CCS definition of mechanism is adopted, policy design should change some elements of the structure of the actual mechanism so that the recurrent pattern of interactions, which is conducive to the outcome of interest, is either changed or addressed in the intended manner.

Based on these two essential lessons, a mechanism-based policy design perspective will improve the capacity of designers to analyze policy tools and programs when policies are under formulation and to better predict the impact on implementation. From this point of view, the variable-oriented perspective 
through which design decisions are often made in which outcomes are modeled using correlations rather than mechanisms, should be replaced with a more finely tuned view of the likely outcome after the designed policy is implemented.

\section{A MECHANISTIC FRAMEWORK FOR POLICY DESIGN: ACTIVATORS, FIRST-ORDER AND SECOND-ORDER MECHANISMS}

By adopting a mechanistic perspective, policy designs can better deal with the multilevel complexity of policy dynamics. By focusing on underlying mechanisms, policy design can be explanatory and predictive, without relying on overreaching theories based on correlative and other forms of logic. Thus, a mechanistic approach can help policy design to better open the "black box" of policy behavior and to better intervene in it.

From this point of view, a mechanistic approach to policy design can facilitate both generalization and prediction and thus better design of policies. Through policy design, different orders of mechanisms can be activated to produce desired outcomes, and different design actions can activate different sequences of mechanisms. Some of these mechanisms are first-order mechanisms, which leverage the expected behavior tendencies of actors to alter their behavior, leading them to perform specific actions that are consistent with the achievement of desired policy outcomes. Others are second-order mechanisms, which are activated to "transform" the initial situation and context of policy-making.

In other words, policy design seeks to change a situation to by adopting policy instruments to activate mechanisms which induce actors to change their behaviors in ways that lead to the emergence of a new transformed situation ( $t 1)$. This type of reasoning translates into policy design through the macro-micro-macro model proposed by Coleman (1990), Hedström and Swedberg (1998) and Hedström and Ylikoski (2010).

Figure 1.1 presents how a policy design mechanism-based approach works.

There are thus three main components of the mechanistic framework of policy design represented in Figure 1.1: activators, first-order mechanisms and second-order mechanisms (Capano and Howlett, 2019).

- "Activators" are not mechanisms: rather, they are "events" or activities that trigger mechanisms, thus activating the causal forces (first-order and second-order mechanisms) through which the behavior of individuals, groups and subsystems is altered to achieve a specific outcome. From a policy design perspective, activators are those policy instruments through which decision-makers set up their policy design to impact on the reality they want to maintain or to change. This does not exclude that mechanistic activation can also be caused by a specific contextual event (generated by 


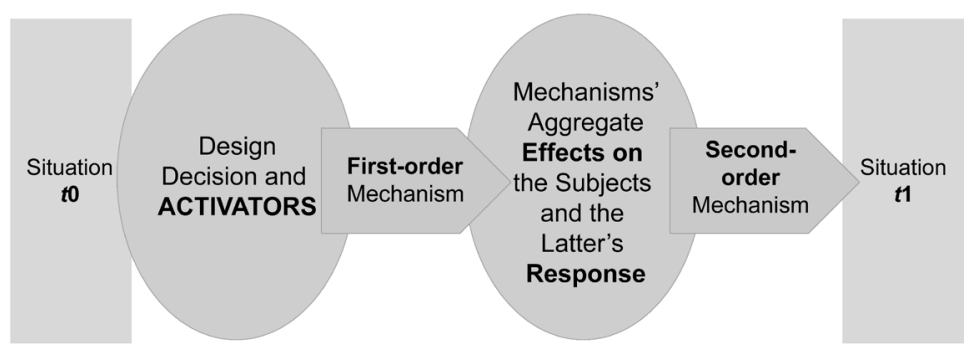

Figure 1.1 The mechanistic process from a policy design perspective

socio-political economic dynamics, or by specific policy actors, like policy entrepreneurs) as well as by the consequence of previous mechanistic processes in a feedback-like process.

- First-order mechanisms are those that directly alter the behavior of individuals and groups to achieve a specific outcome. These mechanisms can be activated by individuals (through actions like the provision of subsidies to trigger enhanced savings behavior), or groups (by extending tax credits for charitable donations, which can lead to the formation of new groups) or to the arrangements of a specific subsystem (for example, by adding or removing new actors and ideas or reinforcing existing ones in a policy network or community).

- Second-order mechanisms are those through which the effects of the first-order mechanisms are aggregated, thus producing a structural or contextual effect. Frequently appearing second-order mechanisms include learning, diffusion transfer, constituency building, adaptive expectations, civic engagement, institutional complementarity, social trust, isomorphism and other similar phenomena. Second-order mechanisms can also be defined as counter-causal mechanisms (counter-mobilization; negative framing; resistance; opportunism) that can impede expected outcomes (Weaver, 2010; Jacobs and Weaver, 2015; Dunlop, 2017).

Thus, Figure 1.1 represents the process through which activators trigger the causal force (first-order mechanisms) through which the behavior of individuals and groups is addressed to achieve a specific outcome. This can be accompanied by the activation of second-order mechanisms that, by reinforcing or changing situation $t 0$, alter the context at point $t$.

Regardless of whether or not second-order mechanisms are consciously activated, after a new policy has been implemented, agents interact with their environment and begin to produce effects that may not have been originally 
intended but can also activate second-order mechanisms. For instance, when a new policy is successful, it may engender learning and diffusion, whether or not this was planned or intended.

For example, in higher education policy, the introduction of national research standards to measure the quality of university research is a design intervention ("activator") through which policy-makers can pursue the improvement of research quality through the activation of the mechanism of institutional competition ("first-order mechanism"). This mechanism is supposed to be quite strong: a significant amount of public funding is allocated through this type of exercise. Theoretically, positive feedback would imply that through competition, the overall national quality of research should increase in the medium run and that through "second-order mechanisms" such as learning or blame avoidance, institutions should institutionalize the new situation $t 1$. It could be the case, however, that because of incoherent design and poor first-order mechanism activation, the new design does not produce the expected behavior and, therefore, the activated competition mechanisms do not trigger the expected second-order ones. This situation has been seen in the UK, for example, where the overall quality of national research has not been improved as much as expected through the introduction of competition for funds. Competition has merely advantaged older, more prestigious institutions (Schafer, 2016), whereas creative, innovative and risky research has been stymied (Talib, 2002; Oancea, 2010). Furthermore, opportunistic behavior in the recruitment (often based on short-term motivations) and distribution of internal power has created significant problems for many schools (Marginson, 2014; McNay, 2015). Thus, in this case, competition (as a first-order mechanism) activated "second-order" counter-causal mechanisms such as opportunism and dissatisfaction, all of which can undermine returns.

This way of reasoning allows us to clearly distinguish between decisional strategies and policy instruments contents (activators) with respect to the causal forces that work behind policy dynamics and to better understand both policy success and failure. Too often, in fact, the content of decisions or the way of deciding has been confused or overlapped with causal mechanisms. For example, very often, the chosen policy instruments (tariffs, exhortation, strong regulations) or the manner of policy design (layering, planning, evidence-based design) are intended to be causal mechanisms in themselves, while rather, they are ways through which causal mechanisms are activated. Furthermore, the distinction between two levels of mechanisms allows a better ordering and comprehension of the process of causation through which policy processes and outcomes develop, and enlightens the complex dynamics that the implementation of any policy design effort has to deal with.

Of course, this is not to say that everything that can be known about policy mechanisms is known, or about which kinds of mechanisms trigger which 
kinds of behavior. The goal of the book is to better understand first-order and second-order mechanisms so these can be better activated through better design.

\section{THE CONTENT OF THE BOOK}

Chapters in the book discuss in more detail the mechanistic logic set out above and use case study and bibliometric methods to explicate the workings of first-order (policy tools, for example) and second-order (policy learning, for example) mechanisms. Various empirical and theoretical aspects offered by the theoretical framework sketched above are explored in detail.

In the first part of the book, additional theoretical insights are offered to better understand why it is important to study mechanisms in/for policy design. This introductory chapter is followed by Evert Lindquist and Adam Wellstead in Chapter 2, who review the most reputed and adopted analytical frameworks of the theories of the policy process: the Multiple Streams Framework, the Advocacy Coalition Framework, Punctuated Equilibrium Theory, Institutional Analysis and Design, Policy Diffusion, Policy Feedback Theory, and Narrative Policy Framework. The authors show that many of these frameworks have considerable overlap in terms of identifying the variables, components, and functions at play in policy-making systems and how they are very loose and often unspecified regarding causal mechanisms. A policy mechanism approach poses a challenge to these frameworks but also points to a way forward. According to Lindquist and Wellstead, deepening our understanding on causal mechanisms may well sharpen our appreciation of what each framework does and cannot do.

In Part II of the book, first-order mechanisms are analyzed in a more fine-grained way through a theoretical contribution and three specific empirical cases. In Chapter 3, Michael Howlett elaborates on a specific category of first-order mechanisms - different from those mechanisms that are targeted to influence individuals' or groups' behaviors - which directly affect policy subsystem or structure and behavior that he defines as "network mechanisms." These types of mechanisms are those affecting the number of types of nodes and links present in a policy community or network rather than individual or group behavior, per se. Network mechanisms are activated mostly by procedural policy tools that directly impact subsystem structural elements nodes and links - by introducing new actors or reconfiguring relationships to affect policy targets and drive policy change. These mechanisms activate mechanisms such as the redefinition of actors' structural positions and policy preferences; promotion of network self-regulation; reframing of the criteria for assessing policy success and failure; and reframing the patterns of actors' interactions and of policy conflict dynamics. 
In Chapter 4, Simone Busetti and Bruno Dente propose an analysis of the mechanisms of the Italian policy for food waste prevention to determine the causes. In particular, two activating measures are analyzed: bureaucratic simplifications for donating food and the possibility to donate food after the best-before date (BBD). These activators are expected to trigger very simple causal mechanisms: bureaucratic simplifications cut the costs of donation and hence make donors increase or start donations. The BBD innovation expands the types of products that can be donated and hence enlarge the set of actions available to donors, who can eventually donate all the surplus that they were previously forced to waste. The analysis highlights how features such as the capacity of donors, the quality of surplus, the recovery skills of charities, and the reputational risks incurred by both actors may affect the effectiveness of these two mechanisms and the implementation of the policy. These elements are not directly under the control of designers, but they are nonetheless fundamental for ensuring that design features trigger successfully a causal mechanism and produce the expected changes of behavior. The conclusion shows how, thanks to a mechanistic analysis, policy designers are not only able to have more realistic understanding of food policy, but also about which kinds of design elements should be further added to overcome possible barriers in successfully activating first-order mechanisms for producing the expected policy outcomes.

In Chapter 5, Altaf Virani and M Ramesh review the experience of publicprivate partnerships (PPPs) in India's healthcare sector, and examine issues of policy coherence, contract design, policy capacity and regulation to identify common sources of failure. Based on the analysis, they highlight five levels of failure: goal definition, instrument selection, calibration, implementation and learning. Thanks to this fine-grained analysis they demonstrate that the policy failure of PPPs in the Indian health system is the result of bad design due to the inability of policy designers to both discern and leverage the key causal mechanisms needed to facilitate actors to behave in ways that are conducive to the achievement of overarching policy goals. Poor design and related implementation problems are based on larger institutional weaknesses in policy capacity, regulation, and interorganizational trust and cooperation, which again affect both the diagnosis and operation of first-order mechanisms and successful policy design.

Maria Tullia Galanti and Sarah Giest end Part II with Chapter 6, devoted to the analyses of how certain actors can advance themselves as policy entrepreneurs and promote the activation of certain first-order mechanisms to lead policy changes. They start from the observation that in the context and the processes that are involved in policy-making, entrepreneurs often play a fundamental role in activating and making mechanisms work. This activation role can take different forms, and involve individual and collective actors at multi- 
ple levels of government - national, regional and local. The chapter focuses on the selection of entrepreneurial strategies in various policy contexts, and how these can affect first-order mechanism activation down the line. The authors use examples at the European level and the Italian national and local contexts to highlight the connection between policy context, entrepreneurial strategies and first-order mechanisms and behavioral changes. Galanti and Giest show how entrepreneurial activities can activate first-order mechanisms like the building of a trustworthy network of actors in support, of commitment in favor of a policy idea, and of the exploitation of windows of opportunities at different institutional levels.

Part III is focused on second-order mechanisms and especially on those related to learning and accountability, notably in the European Union, and on mechanisms for effective public sector reforms. In Chapter 7, Claire A. Dunlop and Claudio M. Radaelli focus their analytical attention on the relationship between policy instruments and mechanisms and how policy-makers and designers learn how this relationship works. They analyze the case of impact assessment (IA) in the European Union as a mechanism expected to trigger second-order learning. IA is an evidence-based instrument adopted by the EU in the context of the evidence-based better regulation strategy. The connection between IA and learning is apparently intuitive: IA should bring evidence to bear on the process of selecting policy options, and therefore assist decision-makers in learning from different types of analysis, dialogue with experts and stakeholders, and open consultation. They identify several types of learning related to the tractability of an issue and the certification of actors associated with it to isolate several specific kinds of learning that can be triggered: epistemic, reflexive, bargaining and hierarchical. These types of learning are activated respectively by teaching through evidence-based rationality, dialogue via participation, exchange through consultation, and monitoring and scrutiny.

Mattia Guidi, in Chapter 8, also uses an EU case to assess the usefulness of a mechanistic approach to policy design, by examining, according to this perspective, EU banking union policy. His research question is quite clear: when a new institutional body is established, how much discretion should the new body enjoy? This choice highlights the trade-off between autonomy (to take advantage of expertise and credibility) and democratic accountability as second-order mechanisms triggered by policy designs. According to his principal-agent framework, EU rules define certain activators that enable political principals to exercise direct or indirect control on some agency. The presence of these first-order mechanisms (and the threat of using them) is expected to trigger accountability (second-order mechanism) on behalf of the agency. Guidi shows how the EU process is not expected to activate strong accountability to the EU decision-makers because of the weakness of the rules 
activating the European banking union together with the lack of political will to make European Central Bank really accountable, again highlighting barriers to the affective activation of policy mechanisms.

Edoardo Ongaro focuses in Chapter 9 on the combination between first- and second-order mechanisms for effective policy design by exploring how the analysis of social mechanisms may enlighten our understanding of policy processes of public sector reform. The chapter develops a selective review of published case studies on the subject and extracts from them a number of social mechanisms that may explain how public sector reform process dynamics operate. These include first-order mechanisms like actor certification; attribution of opportunity and threat; threshold-based behavior; brokerage; appropriation of mobilizing structures; and second-order mechanisms like feedback mechanisms and decreasing or increasing marginal returns. Ongaro argues that contextual and administrative legacies should be combined with a mechanistic approach both for better understanding administrative reform dynamics and for making it more effective.

Part IV is devoted to methods for policy design and to mechanistic analysis of policy outcomes. In Chapter 10, R. Kent Weaver suggests the use of reverse engineering as a second-order mechanism for redesigning policy in his chapter on household savings for retirement in advanced industrial countries. Reverse engineering can help extract information from a more rigorous understanding of interactive social, behavioral and policy processes that can lead to improvements in policy activators for achieving the goals set by governments. Weaver argues that the causal mechanisms affecting individuals' retirement savings decisions are extremely complex, because of context, differences in individual life preferences and the presence of structural barriers (especially resource barriers and the individual perception of uncertainty). In these conditions, the type of activators (more or less constraining) depends on the trade-offs chosen by the decision-maker. Thanks to his analysis, Weaver argues that reverse engineering cannot alone lead to "optimal" choices of instruments and settings given the underlying policy trade-offs and political conflicts in policy design. Thus, Weaver problematizes a mechanistic approach to policy design. In fact, he observes that behavioral responses are often not a single behavior but many, distinctive behaviors, carried out over a long period of time and that mechanistic chains interconnect with other different mechanisms and thus are complex processes. Thus, at least for the analyzed policy field, where the variety of individual preferences could be very high, he suggests that different mechanisms could work according to different targets.

Finally, in Chapter 11, Mallory E. Compton and Paul 't Hart discuss the value of a dynamic and mechanistic perspective to the study of policy success. They elaborate a three-dimensional concept of policy success (programmatic, process, and political performance), and examine how both first- and 
second-order mechanisms can reinforce or work against these dynamics. Developing a typology of policy loops (driven by configurations of first- and second-order mechanisms) they explore, thanks to many empirical illustrations, how such a perspective can not only inform analytical explanations of policy success and failure, but also purposeful attempts by policy actors to work towards their preferred outcomes.

\section{REFERENCES}

Abbott, A. (2007), "Mechanisms and relations," Sociologica, 2(1), 1-22.

Bechtel, W. and A. Abrahamsen (2005), "Explanation: A mechanist alternative," Studies in History and Philosophy of the Biological and Biomedical Sciences, 36(2), 421-41.

Capano, G. (2013), "Policy dynamics and change: The never-ending puzzle," in E. Araral, S. Fritzen and M. Howlett et al. (eds), Handbook of Public Policy, London: Routledge, pp. 451-62.

Capano, G. and M. Howlett (2019), "Causal logics and mechanisms in policy design: How and why adopting a mechanistic perspective can improve policy design," Public Policy and Administration, forthcoming.

Coleman, J.S. (1990), Foundations of Social Theory, Cambridge, MA: The Belknap Press of Harvard University Press.

Dunlop, C.A. (2017), 'Pathologies of policy learning: What are they and how do they contribute to policy failure?', Policy \& Politics, 45(1), 19-37.

Elster, J. (1989), Nuts and Bolts for the Social Sciences, Cambridge, UK: Cambridge University Press.

Fagan, M.B. (2012), "The joint account of mechanistic explanation," Philosophy of Science, 79(4), 448-72.

Falleti, T. and J. Lynch (2009), "Context and causal mechanisms in political analysis," Comparative Political Studies, 4(9), 1143-66.

George, A.L. and A. Bennett (2005), Case Studies and Theory Development, Cambridge, MA: MIT Press.

Gerring, J. (2007), "The mechanismic worldview: Thinking inside the box," British Journal of Political Science, 38(1), 161-79.

Gerring, J. (2010), "Causal mechanisms: Yes but. . .," Comparative Political Studies, 43(11), 1499-526.

Glennan, S. (2011), "Singular and general causal relations: A mechanist perspective," in P.M. Illari, F. Russo and J. Williamson (eds), Causality in the Sciences, Oxford: Oxford University Press, pp. 789-817.

Gross, N. (2009), 'A pragmatist theory of social mechanisms', American Sociological Review, 74(3), 358-79.

Hedström, P. (2008), "Studying mechanisms to strengthen causal inferences in quantitative research," in J. Box-Steffensmeier, H. Brady and D. Collier 
(eds), The Oxford Handbook of Political Methodology, Oxford: Oxford University Press, pp. 319-38.

Hedström, P. and R. Swedberg (1998), Social Mechanisms: An Analytical Approach to Social Theory, Cambridge, UK: Cambridge University Press.

Hedström, P. and P. Ylikoski (2010), "Causal mechanism in the social sciences," Annual Review of Sociology, 36, 49-67.

Jacobs, A. and K. Weaver (2015), "When policies undo themselves: Self-undermining feedback as a source of policy change," Governance, 28(4), 441-57.

Kuorikoski, J. (2009), "Two concepts of mechanism: Componential causal system and abstract form of interaction," International Studies in the Philosophy of Science, 23(2), 143-60.

Mahoney, J. (2001), "Beyond correlational analysis: Recent innovations in theory and method," Sociological Forum, 16(3), 575-93.

Marginson, S. (2014), "Teaching and research in the contemporary university," in V.C.H. Tong (ed.), Geoscience Research and Education: Teaching at Universities, Dordrecht: Springer, pp. 11-18.

Maynitz, R. (2004), "Mechanisms in the analysis of social macro-phenomena," Philosophy of the Social Sciences, 34(2), 237-59.

McNay, I. (2015), "Learning from the UK Research Excellence Framework: Ends and means in research quality assessment, and the reliability of results in education," Higher Education Review, 47(3), 24-47.

Oancea, A. (2010), "Research assessment in the United Kingdom," in UNESCO (ed.), World Social Science Report: Knowledge Divides, Paris: UNESCO Publishing, pp. 259-61.

Schafer, L.O. (2016), "Performance assessment in science and academia: Effects of the RAE/REF on academic life," Working Paper No. 7, Centre for Global Higher Education, UCL Institute of Education.

Schmid, M. (2006), Die Logik mechanismischer Erklärungen [The Logic of Mechanistic Explanations], Wiesbaden: Springer VS Verlag.

Talib, A.A. (2002), "Performance measures and resource allocation: The behavioral consequences of the university research assessment exercise in England," $\mathrm{PhD}$ dissertation, University of Warwick, Warwick Business School.

Tilly, C. (2001), "Mechanisms in political processes," Annual Review of Political Science, 4, 21-41.

Weaver, R.K. (2010), "Paths and forks or chutes and ladders? Negative feedbacks and policy regime change," Journal of Public Policy, 30(2), 137-62. 